\title{
STELLAR COUNTER-ROTATION ALONG THE HUBBLE SEQUENCE: A PROBE FOR GALAXY FORMATION SCENARIOS
}

\author{
F. PRADA \\ Instituto de Astrofísica de Canarias \\ Present address: Instituto de Astronomía, UNAM \\ Apdo. Postal 877, Ensenada, Baja California, C.P. 22830, \\ Mexico \\ AND \\ C.M. GUTIÉRREZ \\ Instituto de Astrofisica de Canarias \\ 38200, La Laguna, Tenerife, Spain
}

\begin{abstract}
We present some preliminary results of an on-going project to investigate the phenomenon of counter-rotation in galaxies. The analysis of two $\mathrm{Sb}$ and two barred galaxies shows the presence of stellar counterrotating components in the central 1 to $3 \mathrm{kpc}$ of each galaxy. This, along with similar structures found in ellipticals and early-type spirals, demonstrate that the counter-rotation extends along the Hubble sequence. The physical origin of these features is briefly discussed stressing the possible relevance of this phenomenon in understanding the general problem of galaxy formation.
\end{abstract}

\section{Introduction}

It is now well established that some elliptical galaxies contain counterrotating cores (Franx \& Illingworth 1988; Rix \& White 1992; Bender et al. 1994), and S0/Sa's can display extended retrograde motions in their discs (Rubin et al. 1992; Rix et al. 1992; Bertola et al. 1996). However very little is known about late type spirals and barred galaxies (see Bertola for a review). The study by Prada et al. (1996) reveals the presence of a retrograde bulge in the nearby Sb galaxy NGC 7331 and no signs of extended counterrotating components have been found in barred systems. This presentation is focussed on some observational results on the stellar counter-rotation in 

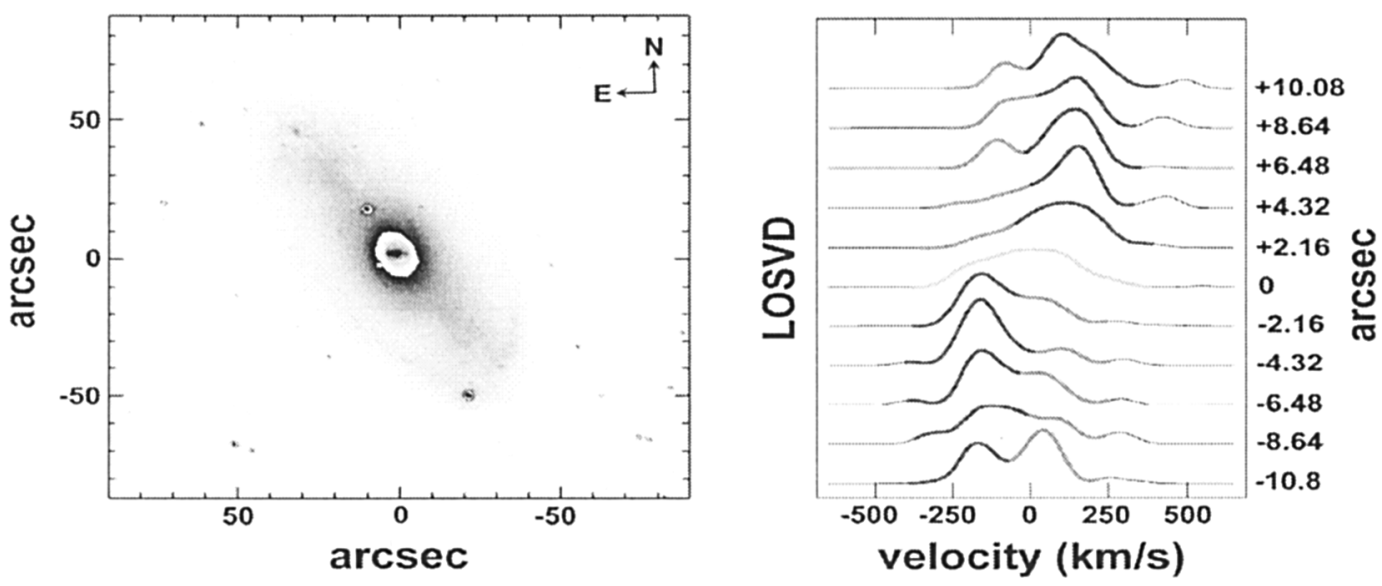

Figure 1. Left: a greyscale I-band image of the barred galaxy NGC5728; Right: the LOSVD along the main bar major axis of NGC 5728

galaxies. The main aims of this project are to study the dynamical properties of retrograde components, to understand the formation mechanism of stellar counter-rotating components along the Hubble sequence.

\section{The observations, the algorithm and the parametrization of LOSVD}

For a sample of two Sb galaxies (NGC 7331, NGC 2841) and two barred galaxies (NGC $5728 \mathrm{SBa}$; NGC $5005 \mathrm{SBbc}$ ) long-slit observations were performed with the ISIS spectrograph on the $4.2 \mathrm{~m}$ WHT telescope at La Palma. The resolution was 25 and $50 \mathrm{~km} \mathrm{~s}^{-1}$ (depending of the object observed) in the spectral region around the CaII IR triplet ( 8550 $\AA)$. The slit was placed along the major axis of the spirals and along the main bar major axis in the barred galaxies. More details of the observations and data reduction will be presented in future publications. The stellar line-of-sight velocity distributions (LOSVD) were determined from the long-slit CaII triplet absorption spectra by means of a two-dimensional unresolved Gaussian decomposition algorithm. This is the method used to determine the stellar kinematical structure of NGC 7331 (see Prada et al. 1996). Details about the algorithm can be found in that paper and in Prada et al. 1998.

The LOSVDs of these galaxies clearly shows two distinct components that can be well-parametrized by two Gaussians (see Fig. 1). The parameters of each Gaussian gives for each position in the galaxy recession velocities, dispersions and relative flux of the two components. The comparison of the relative flux with the photometry of the galaxy allows us to identify kinematical with photometric structures (see Figure 2). 


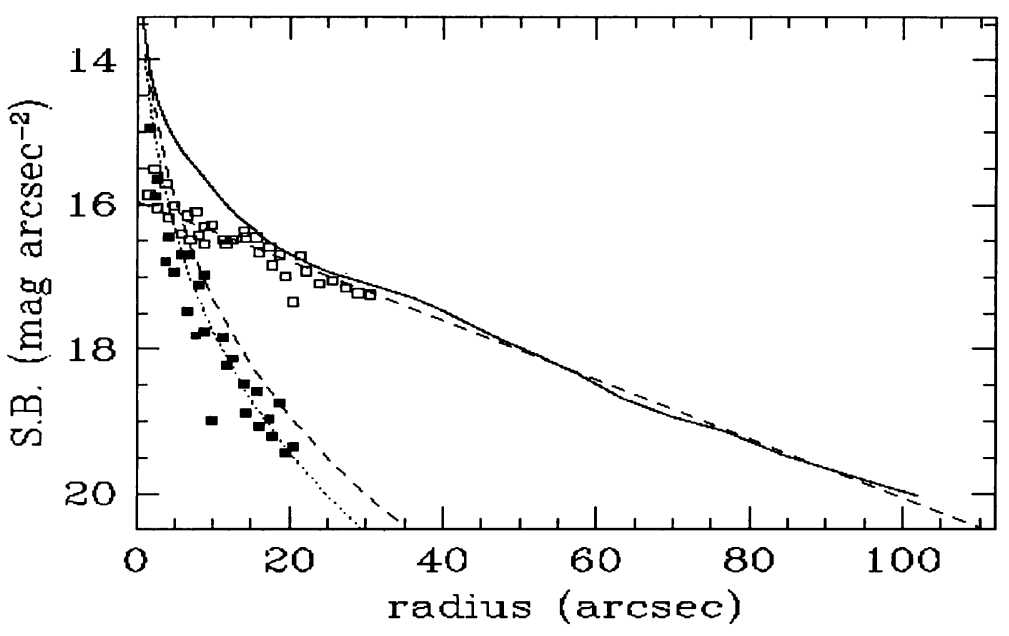

Figure 2. The major axis surface brightness profile of NGC 7331. The profile has been decomposed into an exponential disk and an $\mathrm{r}^{1 / 4}$ component. We have also plotted the relative fluxes of the direct component (open symbols) and the counter-rotating component (dark symbols). Notice that the points of the retrograde component follows well the profile of the bulge and the direct component data agree with the disk profile.

\section{Discussion and conclusions}

The four galaxies presented here show extended counter-rotation in the central 1 to $3 \mathrm{kpc}$. The two $\mathrm{Sb}$ galaxies show a boxiness and a position angle twist of $\sim 10-15^{\circ}$ which makes very likely that we are seeing a triaxial body in projection (e.g. Statler 1991). The retrograde components can be fairly warm $(v / \sigma \sim 0.5-1)$ and boxy. They can be associated with the bulge (e.g. NGC 7331) and/or with the centre of a non-axisymmetric structure in the galaxy (e.g. NGC 5728). These results along with the previous work on the ellipticals and early-type spirals demonstrate that the counter-rotation is a phenomenon common along the Hubble sequence. Having established this, next question is to find out the origin of this. Two basic mechanisms have been proposed; the first involves accretion or mergers. For instance the counter-rotating cores in elliptical galaxies have been explained as a merger with a small dwarf galaxy (e.g. Balcells \& Quinn 1990), while in this scenario the counter-rotating disks could be produced by accretion of a satellite with a retrograde orbit, however numerical simulations show that in most of the cases this will heat up the disk (see Thakar \& Ryden 1996). The second kind of mechanisms involves only the internal evolution of the galaxy. This includes the process of galaxy formation itself from primordial clouds. The problem here is that it requires an efficient mechanism to change the angular momentum drastically as a function of radius. Dynami- 
cal instabilities like the disruption of a bar or the distribution of retrograde orbits in the bar (e.g. Wozniak \& Pfenniger 1997), infall of gas (Thakar \& Ryden 1996) and structures formed later from processed gas (Bender \& Surma 1992) have been proposed as well. These scenarios have to explain why the relative fraction of rotating/counter-rotating stars in galaxies like NGC 4550 and NGC 7217 is so constant.

Finally, we would like to finish enphasizing that:

1/ The phenomenon of stellar counter-rotation seems to be present throughout the Hubble sequence.

$2 /$ These retrograde components display a wide variety of dynamical properties and morphology.

3 / The physical origin of counter-rotation remains unclear, being needed better observations (2D spectroscopy), statistics, and more plausible models which can form such retrograde features along the Hubble sequence.

We are grateful to Tim de Zeeuw for discussions and useful comments on the text. This research is based on observations obtained at the WHT, operated by the Isaac Newton Group of telescopes at the Observatorio del Roque de los Muchachos at La Palma of the Instituto de Astrofísica de Canarias.

\section{References}

Balcells M., \& Quinn, P. J., (1990), ApJ, 361, 381

Bender, R., \& Surma, P., (1992), $A \& A, \mathbf{2 5 8}, 250$

Bender, R., Saglia, R. P., \& Gerhard, O. E. (1994), MNRAS, 269, 785

Bertola, F., (1997), this meeting

Bertola, F., Cinzano, P., Corsini, E. M., Pizzella, A., Persic, M., \& Salucci, P. (1996), $A p J$, 458, L67

Franx, M., \& Illingworth, G., (1988), ApJ, 327, L55

Prada, F., Gutiérrez, C. M., Peletier, R. F., \& McKeith, C.D., (1996), ApJ, 463, L9

Prada, F., Gutiérrez, C. M., \& McKeith, C. D. 1998 ApJ (in press)

Rix, H.-W., \& White, S. D. M., (1992), MNRAS, 254, 389

Rix, H.-W., Franx, M., Fisher, D., \& Illingworth, G., (1992), ApJ, 400, L5

Rubin, V., Graham, J., \& Kenney, J., (1992) , ApJ, 394, L9

Statler, T. S. (1991), ApJ, 102, 882

Thakar. A, \& Ryden. B., (1996), ApJ, 461, 55

Wozniak, H. \& Pfenniger, D., (1997) $A \& A$, 317, 14 